\title{
Laser structuring for improved battery performance
}

Wilhelm Pfleging, Melanie Mangang, Yijing Zheng, and Peter Smyrek

New material architectures and electrode surface topographies are used in high-power batteries to improve battery lifetime and cell stability.

Within the last two decades, lithium-ion batteries (LIBs) have emerged as the power source of choice in the high-performance rechargeable battery market. ${ }^{1,2}$ LIBs are high-capacity batteries that are able to store electricity converted from green energy sources (e.g., solar and wind power), and they can act as energy sources in pollution-free electric vehicles. Nevertheless, there are several drawbacks to state-of-the-art LIBs, including high production costs, short battery lifetimes, safety issues, and time-consuming charging periods. The main issue in cell production is the electrolyte wetting of LIBs, which is realized through time- and cost-consuming vacuum and storage processes at elevated temperatures. Insufficient wetting of electrodes results in a production failure rate, accompanied by a reduced cell capacity and cell lifetime.

The development of 3D electrode architectures in LIBs is a relatively new approach for overcoming the problems related to battery performance (e.g., power losses or high interelectrode ohmic resistances ${ }^{3}$ ) and mechanical degradation ${ }^{4}$ during battery operation. 3D batteries can be used to achieve large areal energy capacities, while simultaneously maintaining high power densities. A common approach is 3D structuring of the electrode substrate (the 'current collector') before deposition of the thin-film electrode. Unfortunately, this method is in a very early stage of development for model electrodes in thin-film microbatteries. Furthermore, it is generally not feasible for up-scaling to thick-film composite electrodes or for large electrode footprint areas.

At the Karlsruhe Institute of Technology, we have thus developed a new technical approach for the generation of 3D electrode designs that can be applied to all types of LIBs (i.e., thin-film batteries, as well as high-energy and high-power LIBs). ${ }^{5}$ In this approach, for the first time, we have applied laser-assisted processing to the active electrode material itself. For this
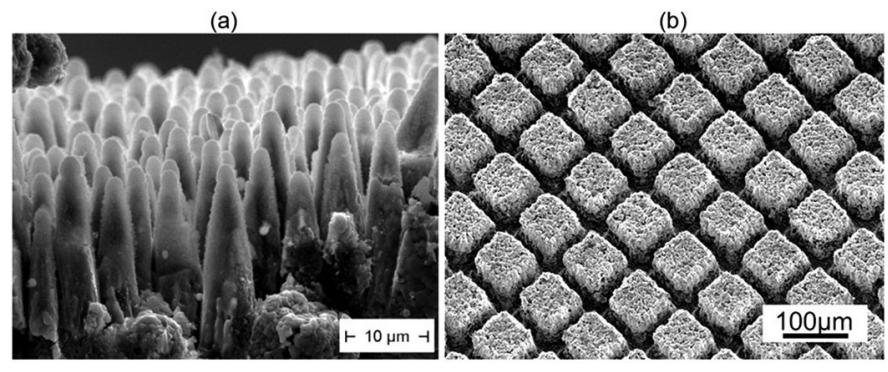

Figure 1. Scanning electron microscope images of laser-generated microstructures in composite electrode materials. (a) Self-organized microstructures (produced with the use of an excimer laser) and (b) micro-pillars obtained from direct laser structuring with an ultrafast (femtosecond) laser.

purpose, we established different types of laser processing for increasing the active surface area, ${ }^{6-8}$ i.e., 'laser-assisted selforganizing structuring' and 'direct structuring' of electrodes. The first of these processes can be applied to thin films and thickfilm electrodes that have small electrode footprint areas (coin cells). The second process is suitable for small and large electrode footprint areas (pouch cells).

We used excimer laser ablation at a wavelength of $248 \mathrm{~nm}$ to produce self-organized surface structures—see Figure 1(a)—on lithium cobalt oxide and lithium nickel manganese cobalt oxide (NMC) thick- and thin-film electrodes. This self-organized structuring process ${ }^{8-10}$ is possible because of selective material ablation and subsequent material re-deposition. We were able to avoid material loss during laser patterning, and we found that the active surface area could be increased by a factor of 10 . We also used direct laser structuring - either with a 200ns fiber laser or an ultrafast fiber laser (380fs) - to form 3D microstructures, as shown in Figure 1(b). We conducted this structuring process under ambient air conditions, and the ablated material was removed through an exhaust.

The nanosecond and ultrafast laser structuring methods that we have developed for the formation of capillary

Continued on next page 
micro-structures in thick-film tape-cast electrodes give rise to a tremendous acceleration and homogeneity of electrolyte wetting (see Figure 2). We find that an appropriate structure design and complete removal of the electrode material from the ablation zone delivers the most efficient capillary transport. ${ }^{11}$ Nanosecond laser ablation, however, is not appropriate for all

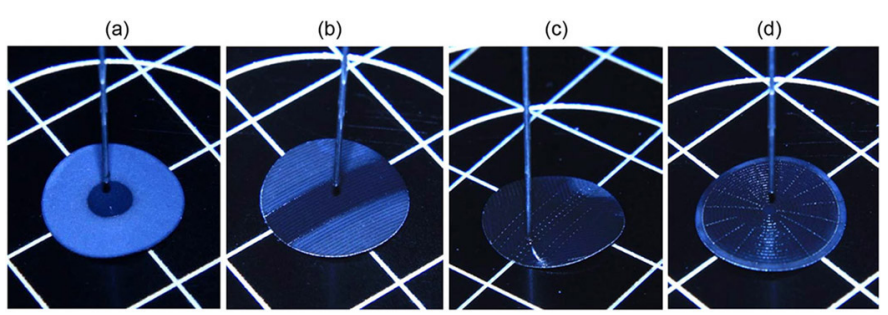

Figure 2. Single-drop-electrolyte $(3 \mu \mathrm{l})$ wetting for (a) unstructured and $(b)$ to $(d)$ laser-structured thick-film electrodes.
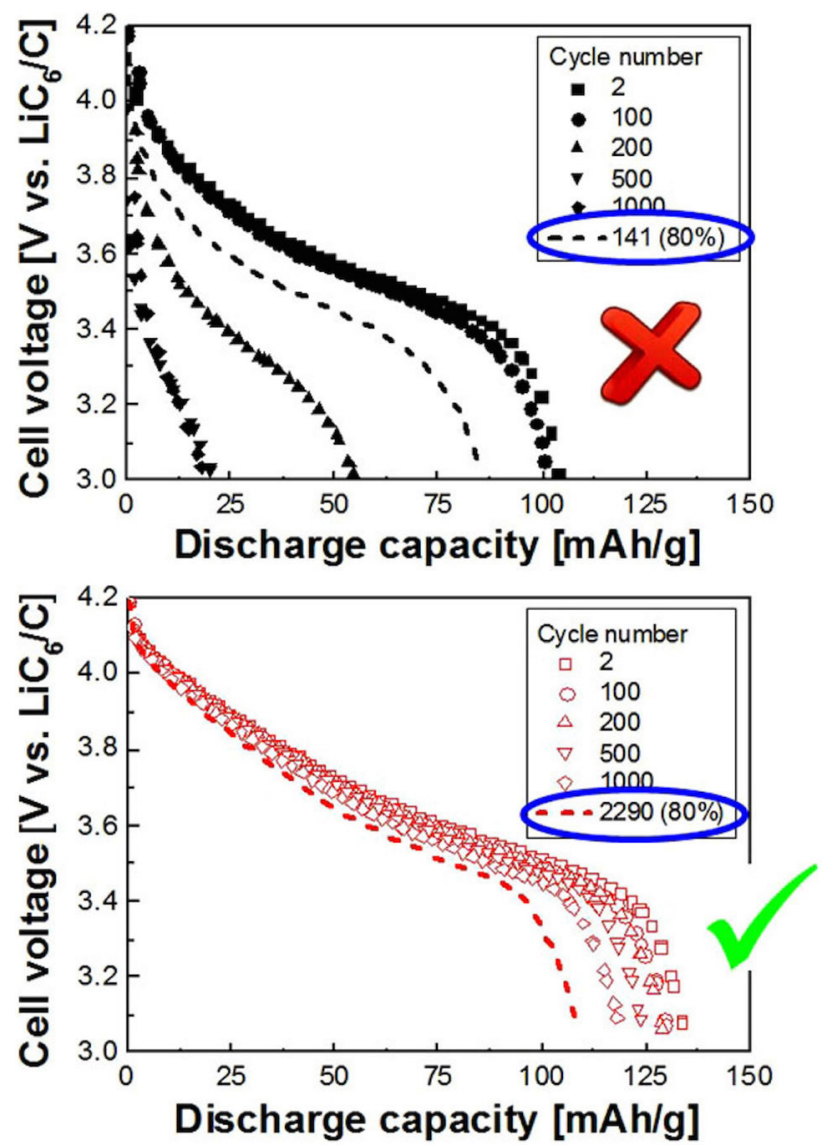

Figure 3. Cell voltage versus discharge capacity for pouch cells that have unstructured (top) and laser-structured (bottom) lithium nickel manganese cobalt oxide electrodes (without previous storage). ${ }^{11}$ Li: Lithium. C: Carbon. types of electrode materials. For example, lithium iron phosphate changes the chemical composition in the nanosecondlaser-induced heat-affected zone (this can be avoided with the use of cold ultrafast laser ablation). Furthermore, ultrafast laser ablation has an increased ablation efficiency compared with nanosecond laser ablation. ${ }^{12}$ The loss of active material can also be reduced from $20 \%$ to values that are below $5 \%{ }^{13}$

For LIBs that have structured NMC electrodes, we find that the $80 \%$ capacity limit of the initial discharge capacity is reached after 2290 cycles (see Figure 3). For LIBs with unstructured electrodes, however, our results indicate that the cell lifetime is reached after 141 cycles (without cost- and time-consuming storage procedures). This significant increase in battery lifetime is caused by efficient and instantaneous liquid electrolyte transport that is forced by the laser-generated micro-capillary structures.

In summary, we have developed new laser-assisted processing methods that can increase the active surface area of electrodes in lithium-ion batteries. The laser-generated capillary structures we form in electrode materials increase cell reliability and shorten battery production times. Improved cycle lifetimes and increased capacity retention also mean that high-power batteries in second-life applications become a possibility. Our future work will be focused on tuning each type of active material for high-power and high-energy batteries, as well as for future battery concepts. In addition, process up-scaling for large electrode footprint areas will contribute to significantly decreased manufacturing costs, while maintaining and improving overall battery performance and safety.

The authors are grateful for support of laser processing from the Karlsruhe Nano Micro Facility (a Helmholtz research infrastructure at the Karlsruhe Institute of Technology).

\section{Author Information}

Wilhelm Pfleging, Yijing Zheng, and Peter Smyrek Applied Materials Physics (IAM-AWP) and Karlsruhe Nano Micro Facility (KNMF)

Karlsruhe Institute of Technology

Karlsruhe, Germany

Wilhelm Pfleging is head of the Laser Materials Processing group. His application-oriented research is focused on the development of laser-assisted processes for advanced LIBs. 


\section{Melanie Mangang}

\section{IAM-AWP}

Karlsruhe Institute of Technology

Karlsruhe, Germany

\section{References}

1. J. B. Goodenough and Y. Kim, Challenges for rechargeable Li batteries, Chem. Mater. 22, pp. 587-603, 2010.

2. B. Scrosati and J. Garche, Lithium batteries: status, prospects, and future, J. Power Sources 195, pp. 2419-2430, 2010.

3. J. W. Long, B. Dunn, D. R. Rolison, and H. S. White, Three-dimensional battery architectures, Chem. Rev. 104, pp. 4463-4492, 2004.

4. R. Kohler, H. Besser, M. Hagen, J. Ye, C. Ziebert, S. Ulrich, J. Proell, and W. Pfleging, Laser micro-structuring of magnetron-sputtered $\mathrm{SnO}_{x}$ thin films as anode material for lithium ion batteries, Microsyst. Technol. 17, pp. 225-232, 2011.

5. W. Pfleging, P. Smyrek, M. Mangang, Y. Zheng, and J. Pröll, Laser processes and analytics for high power 3D battery materials, Proc. SPIE 9740, p. 974013, 2016.

doi:10.1117/12.2212041

6. J. Pröll, H. Kim, A. Piqué, H. J. Seifert, and W. Pfleging, Laser-printing and femtosecond-laser structuring of $\mathrm{LiMn}_{2} \mathrm{O}_{4}$ composite cathodes for Li-ion microbatteries, J. Power Sources 255, pp. 116-124, 2014.

7. J. H. Park, R. Kohler, W. Pfleging, W. Choi, H. J. Seifert, and J. K. Lee, Electrochemical behavior of a laser microstructured fluorine doped tin oxide anode layer with a plasma pretreatment for 3D battery systems, RSC Adv. 4, pp. 4247-4252, 2014.

8. R. Kohler, J. Proell, M. Bruns, S. Ulrich, H. J. Seifert, and W. Pfleging, Conical surface structures on model thin-film electrodes and tape-cast electrode materials for lithiumion batteries, Appl. Phys. A 112, pp. 77-85, 2013.

9. B. Ketterer, H. Vasilchina, K. Seemann, S. Ulrich, H. Besser, W. Pfleging, T. Kaiser, and C. Adelhelm, Development of high power density cathode materials for Li-ion batteries, Int'1 J. Mater. Res. 99, pp. 1171-1176, 2008.

10. R. Kohler, P. Smyrek, S. Ulrich, M. Bruns, V. Trouillet, and W. Pfleging, Patterning and annealing of nanocrystalline $\mathrm{LiCoO}_{2}$ thin films, J. Optoelectron. Adv. Mater. 12, pp. 547-552, 2010.

11. W. Pfleging and J. Pröll, A new approach for rapid electrolyte wetting in tape cast electrodes for lithium-ion batteries, J. Mater. Chem. A 2, pp. 14918-14926, 2014.

12. M. Mangang, H. J. Seifert, and W. Pfleging, Influence of laser pulse duration on the electrochemical performance of laser structured $\mathrm{LiFePO}_{4}$ composite electrodes, J. Power Sources 304, pp. 24-32, 2016.

13. P. Smyrek, J. Pröll, H. J. Seifert, and W. Pfleging, Laser-induced breakdown spectroscopy of laser-structured $\mathrm{Li}(\mathrm{NiMnCo}) \mathrm{O}_{2}$ electrodes for lithium-ion batteries, J. Electrochem. Soc. 163, pp. A19-A26, 2016. 